TAUP 2760-04

15 March, 2004

\title{
Stark Effect in Lax-Phillips Scattering Theory
}

\author{
Tamar Ben Ari ${ }^{1}$ L.P. Horwitz ${ }^{1,2}$ \\ ${ }^{1}$ Department of Physics, Bar Ilan University \\ Ramat Gan 52900, Israel \\ ${ }^{2}$ School of Physics, Tel Aviv University \\ Ramat Aviv 69978, Israel
}

\begin{abstract}
The scattering theory of Lax and Phillips, originally developed to describe resonances associated with classical wave equations, has been recently extended to apply as well to the case of the Schrödinger equation in the case that the wave operators for the corresponding Lax-Phillips theory exist. It is known that the bound state levels of an atom become resonances (spectral enhancements) in the continuum in the presence of an electric field (on all space) in the quantum mechanical Hilbert space. Such resonances appear as states in the extended Lax-Phillips Hilbert space. We show that for a simple version of the Stark effect, these states can be explicitly computed, and exhibit the (necessarily) semigroup property of decay in time. The widths and location of the resonances are those given by the poles of the resolvent of the standard quantum mechanical form.
\end{abstract}




\section{Introduction}

The description commonly used for an unstable system is that of Wigner and Weisskopf $^{1}$. They assumed that the unstable system is represented by a vector, say $\phi$, in the Hilbert space of states, and that the Hamiltonian evolution of this state permits the development of other components which represent the "decayed" system. One may assume a Hamiltonian $H_{0}$ for which $\phi$ is an eigenstate, and the full Hamiltonian is constructed by adding a pertubation $V$ which induces transitions from this state. The basic assumption of their physical model is that these transitions carry the state of the system from that of some specific system to a state in the continuous spectrum which contains the decay products. For example, one may think of an atom in an excited state as the unstable system; in the absence of electromagnetic interaction, this state would be a stable bound state. A term added to the Hamiltonian corresponding to electromagnetic interaction induces a transition from this state, and the square of the corresponding transition amplitudes, which may be generally computed perturbatively, give the probability for decay. This very standard technique is rigorously correct for reversible quantum transitions, according to the laws of quantum theory. When applied to the decay of an unstable system, however, for which the evolution is irreversible, it consitutes an approximation which may be inadequate. In the following we discuss some of the difficulties of the application of the Wigner-Weisskopf method to the treatment of irreversible phenomena, such as particle decay, and in Section 3 we describe the general structure of the Lax-Phillips theory, which yields an exact semigroup evolution and appears to be a more appropriate description of such phenomena. In Section 2, we apply the Wigner-Weisskopf theory to a simple model for the Stark effect, for comparison, and work out the Lax-Phillips theory for this model in Section 4. Some of the calculations in Section 2 coincide with those needed in Section 4; the approximate pole approximation decay law of the Wigner-Weisskopf theory contains exactly the same pole as the singularity of the Lax-Phillips $S$ matrix, so that the decay laws are (approximately) the same, but, as we shall see, there are important differences in that the Lax-Phillips treatment leads to an exact semigroup and contains information not accessible in the Wigner-Weisskopf method.

One easily sees that the Wigner-Weisskopf procedure cannot yield a semigroup property of evolution. The "survival amplitude" for such a state is given by

$$
A(t)=\left(\phi, e^{-i H t} \phi\right),
$$

and the survival probability is $|A(t)|^{2}$. One observes, in general, that decaying systems are characterized by exponential decay in time. However, the time derivative of $|A(t)|^{2}$ at $t=0$ is

$$
\begin{aligned}
\left.\frac{d}{d t}|A(t)|^{2}\right|_{t=0} & =-i(\phi, H \phi)+i(H \phi, \phi) \\
& =0
\end{aligned}
$$

where the last equality follows from the self-adjointness of $H$. Hence the probability curve must arrive at the origin with zero slope, and cannot be exponential. Generally, this deviation from exponential occurs only for very short times ${ }^{2,3}$, e.g., for a weak decay with lifetime of the order of $10^{-10}-10^{-8}$ seconds, this short time may be of order $10^{-22}$ seconds. The dominating exponential behavior for intermediate times can well account for the data 
in many such cases of single channel decay. For multichannel problems (many types of final states), for example, the two channel neutral K-meson decay, the lack of semigroup property may lead to disagreement with experiment.

To see this (and to see as well the origin of the exponential part of the decay), let us examine briefly the two channel case. In this case, the probability of decay is given by

$$
\left.P=\sum_{j} \int d \lambda_{j}\left|<\lambda_{j}\right| e^{-i H t} \phi\right)\left.\right|^{2},
$$

where $\left\{\lambda_{j}\right\}$ corresponds to the continuous spectrum of the "final" states, and $\phi$ is an arbitrary state in the two dimensional subspace of initial states (e.g., $K^{0}, \bar{K}^{0}$ ). This can be written alternatively, since the evolution is unitary, as

$$
P=1-\sum_{j}\left|\left(\phi_{j}, e^{-i H t} \phi\right)\right|^{2},
$$

where $\left\{\phi_{j}\right\}$ spans the subspace. Since one can always express $\phi$ as a linear superposition of the $\left\{\phi_{j}\right\}$, it is sufficient to study the quantities

$$
\begin{aligned}
A_{i j} & =\left(\phi_{i}, e^{-i H t} \phi_{j}\right) \\
& =\frac{1}{2 \pi i} \int_{C} e^{-i z t}\left(\phi_{i}, \frac{1}{z-H} \phi_{j}\right) d z \\
& =\frac{1}{2 \pi i} \int_{C} e^{-i z t}\left(\frac{1}{z-W(z)}\right)_{i j} d z,
\end{aligned}
$$

where $W(z)$ is a $2 \times 2$ matrix defined by the second equality, $C$ is a contour for the inverse Laplace transform, and the quantity $(z-H)^{-1}$ is the resolvent operator associated with the Hamiltonian $H$ (defined for $z$ in the upper half plane). The contour $C$ runs above the real line from $+\infty$ to $-\infty$. Almost every $2 \times 2$ matrix has a decomposition ${ }^{4}$ of spectral type

$$
W(z)=z_{1}(z) Q_{1}(z)+z_{2}(z) Q_{2}(z),
$$

where the $Q_{i}(z)$ are constructed from the right and left eigenvectors of $W(z)$, and satisfy $Q_{i}^{2}=Q_{i}, Q_{i} Q_{j}=0, i \neq j$. The resolvent kernel is regular in the upper half plane, but its analytic continuation through the cut induced by the continuous spectrum of $H$ may have poles (or, in principle, other singularities). For small perturbations, one expects that the bound states in $H_{0}$ for a model for which $H=H_{0}+V$ would move slightly into this region, which we shall call the second Riemann sheet ${ }^{4}$. Poles would appear as zeros in either $z-z_{1}(z)$ or in $z-z_{2}(z)$, at points $z_{a}$ or $z_{b}$. Let us assume that the eigenvalues are ordered so that one pole appears at $z_{a}$ such that $z_{a}-z_{1}\left(z_{a}\right)=0$ and the second at $z_{b}$ such that $z_{b}-z_{1}\left(z_{b}\right)=0$. In distorting the contour below the real axis across the cut into the second sheet, these pole contributions may dominate the intermediate time behavior of the amplitude (the very short time behavior is controlled by the Taylor expansion at small $t)^{3}$. For semibounded spectrum, in $(0, \infty)$, the long time behavior is controlled by 
the contribution at the branch point, and goes as $t^{-n}$, where $n$ is the dimensionality of space. The resulting pole approximation is then

$$
A_{i j}(t) \cong e^{-i z_{a} t} Q_{1}\left(z_{a}\right)+e^{-i z_{b} t} Q_{2}\left(z_{b}\right)
$$

where we have also approximated the factor in the residue arising from the derivative of the denominator in the neighborhood of the singularity by unity.

For the single channel case, one sees the emergence of the simple exponential behavior for intermediate times. However, for the two channel (or more) case, we see that the matrix $A_{i j}(t)$ does not satisfy the semigroup property

$$
A(t) A\left(t^{\prime}\right)=A\left(t+t^{\prime}\right)
$$

because, in general (in the absence of $\mathrm{CP}$ conservation), $Q_{1}\left(z_{a}\right) Q_{2}\left(z_{b}\right) \neq 0$ (in case $W(z)$ is independent of $z$, these matrices would be, of course, orthogonal).

One may think of this semigroup property as a requirement for the evolution of an irreversible system ${ }^{5}$; this follows from arguing that the evolution on a Hilbert space must be composable (in the sense that a product of evolutions must be equivalent to a single evolution over the total interval) for a sequence of $t$ 's that are positive, since the system carries no dependence on its past, but the inverse does not exist. Indeed, experiments carried out at Fermilab ${ }^{6}$ show that the phenomenological model of Lee, Oehme, Yang and $\mathrm{Wu}^{7}$ is an extremely accurate representation. This model assumes that the evolution in the two dimensional subspace is given by $\exp \left(-i H_{2 \times 2} t\right)$, where $H_{2 \times 2}$ is a $2 \times 2$ nonHermitian matrix. In this case, the matrix $H_{2 \times 2}$, independent of $z$, replaces $W(z)$ in the the spectral decomposition (1.5); the pole residues are orthogonal, and one obtains the exact semigroup law. Using a Lee-Friedrichs model $^{4}$ to estimate the errors, one finds that the non-orthogonality of $Q\left(z_{a}\right)$ and $Q\left(z_{b}\right)$ induces errors that are larger than the experimental errors in regeneration experiments and in the interference in the exit beam ${ }^{8}$. We infer from these results that for irreversible transitions (of the type occurring in particle decay), one should have the semigroup property.

The theory of scattering developed in 1967 by Lax and Phillips ${ }^{9}$ for application to classical wave equations has the property that resonance decay satisfies exactly the semigroup property. It is also true that, in that theory, there is a well-defined state in the Hilbert space corresponding to the resonance.

This theory has been developed and methods found to apply it to resonances in the quantum theory ${ }^{10-14}$. In the Wigner-Weisskopf method, the resonance is represented only as a pole in the complex plane. Attempts have been made to extend the Hilbert space to the so-called rigged Hilbert space ${ }^{15}$ (Gel'fand triple constuction), in which exact exponential decay occurs, but the "eigenstate" of the resonance, in that case, lies in a Banach space that is not generally a Hilbert space, so that scalar products and expectation values are not defined. The definition of the wave function of the resonance carrying information on its size, shape and momentum distribution could be important in many applications, for example, for the resonance in a quantum dot or a small metallic particle ${ }^{16}$.

It is most straightforward to apply these methods to problems in which the corresponding Hamiltonian of the standard theory has spectrum $-\infty$ to $+\infty$. One such example is 
the relativistic quantum theory ${ }^{17}$, where the unperturbed Hamiltonian has a form proportional to $E^{2}-p^{2}$; the Lax-Phillips theory for such a problem has been worked out ${ }^{18}$. For the non-relativistic case, the spectrum of the Hamiltonian is generally bounded from below, and further studies are being carried out in order to be able to apply the theory in these cases as well ${ }^{14}$. However, the non-relativistic Stark effect is a case in which the non-relativistic problem has a Hamiltonian unbounded from below. We shall study this problem in detail in this paper, and show that one finds semigroup behavior for the decay of the Stark induced resonances; the wave functions for the resonant states are also explicity worked out. In the following we study a simple model, of the type of Friedrichs and Rejto ${ }^{19}$, for the Stark effect, in the framework of Wigner and Weisskopf, and then imbed the results into the more general framework of Lax and Phillips. We shall review the content of the Lax Phillips theory in this later section. 


\section{Wigner-Weisskopf Analysis of a Stark Model}

The potential for the Stark effect problem, of the form $-E x$, is unbounded (on the full space; we shall work in one space dimension). For a model of the form ${ }^{19}$

$$
H=-E x+\lambda P_{0},
$$

where $\lambda$ is real and $P_{0}$ is a rank one projection operator, it will be convenient to consider $-E x \equiv H_{0}$ as the unperturbed Hamiltonian and the second term, $\lambda P_{0} \equiv V$ as the perturbation (the resolvent is, of course unaffected by this choice, but the form of the perturbation theory is very different). We study in this section the Wigner-Weisskopf description of the resonance, and in the next section, imbed this analysis in the Lax-Phillips Hilbert space.

Let us choose for $P_{0}$ the form

$$
<x\left|P_{0}\right| x^{\prime}>=\left(\frac{2}{\pi}\right)^{\frac{1}{2}} e^{-\left(x^{2}+x^{\prime 2}\right)}
$$

The resolvent satisfies the identity (second resolvent equation)

$$
G=G_{0}+G_{0} V G,
$$

where, as above, $G=(z-H)^{-1}$, and $G_{0}=\left(z-H_{0}\right)^{-1}$, defined for $z$ in the upper half plane, where $H_{0}=-E x$. The $x, x^{\prime}$ matrix element of $G$ is therefore

$$
\left\langle x|G| x^{\prime}\right\rangle=\frac{1}{z+E x} \delta\left(x-x^{\prime}\right)+\frac{1}{z+E x} \lambda \int_{-\infty}^{\infty}\left\langle x\left|P_{0}\right| x^{\prime \prime}\right\rangle\left\langle x^{\prime \prime}|G| x^{\prime}\right\rangle d x^{\prime \prime} .
$$

Let us define

$$
f\left(z, x^{\prime}\right)=\int d x^{\prime \prime} e^{-x^{\prime \prime 2}}\left\langle x^{\prime \prime}|G| x^{\prime}\right\rangle d x^{\prime \prime} .
$$

It then follows from (2.4) that

$$
f\left(z, x^{\prime}\right)=\int d x \frac{e^{-x^{2}}}{z+E x}+\lambda \int d x \frac{e^{-x^{2}}}{z+E x} \sqrt{\frac{2}{\pi}} e^{-\left(x^{2}+x^{\prime \prime 2}\right)}\left\langle x^{\prime \prime}|G| x^{\prime}\right\rangle d x^{\prime \prime} .
$$

One can write this as

$$
f\left(z, x^{\prime}\right)=\frac{e^{-x^{\prime 2}}}{z+E x^{\prime}}+\lambda \sqrt{\frac{2}{\pi}} F(z) f\left(z, x^{\prime}\right)
$$

or

$$
f\left(z, x^{\prime}\right)=\frac{1}{1-\lambda \sqrt{\frac{2}{\pi}} F(z)} \frac{e^{-x^{\prime 2}}}{z+E x^{\prime}},
$$

where

$$
F(z)=\int d x \frac{e^{-2 x^{2}}}{z+E x}=\frac{i \pi}{E} e^{-\frac{2 z^{2}}{E^{2}}} \operatorname{erfc}\left[i \sqrt{2} \frac{z}{E}\right] .
$$


Returning to Eq. (2.4), we see that

$$
\begin{aligned}
\left\langle x|G(z)| x^{\prime}\right\rangle & =\frac{1}{z+E x} \delta\left(x-x^{\prime}\right)+\frac{1}{z+E x} \lambda \sqrt{\frac{2}{\pi} e^{-x^{2}} f\left(z, x^{\prime}\right)} \\
& =\frac{1}{z+E x} \delta\left(x-x^{\prime}\right)+\lambda \sqrt{\frac{2}{\pi}} \frac{e^{-x^{2}} e^{-x^{\prime 2}}}{(z+E x)\left(z+E x^{\prime}\right)} \frac{1}{1-\lambda \sqrt{\frac{2}{\pi}} F(z)} .
\end{aligned}
$$

We now wish to approximate the time behavior of the survival amplitude. As in Eq.(1.4), the time dependence of the survival amplitude (for one channel) is given by

$$
A(t)=\frac{1}{2 \pi i} \int_{C}(\varphi|G(z)| \varphi) e^{-i z t} .
$$

The contour $C$ corresponds to a line running in the complex energy plane from right to left slightly above the real axis. The matrix element $(\varphi|G(z)| \varphi)$ is analytic in the upper half-plane. We can shift this line continuously and differentiably through the real axis into the lower half plane, provided that the contribution of the vertical pieces at $\pm \infty$ vanish. It is clear from (2.7) and (2.8) that this is true for the part of the vertical integrations that lie in the upper half plane. To write the integrand along the new curve below the axis, we must analytically continue $F(z)$. To do this, consider (for $\xi$ real)

$$
\begin{aligned}
F(\xi+i \epsilon)-F(\xi-i \epsilon) & =\int_{-\infty}^{\infty} d x e^{-2 x^{2}}\left\{\frac{1}{\xi+i \epsilon+E x}-\frac{1}{\xi-i \epsilon+E x}\right\} \\
& =-2 \pi i \int d x e^{-2 x^{2}} \delta(\xi+E x) \\
& =-\frac{2 \pi i}{E} e^{-\frac{2 \xi^{2}}{E^{2}}}
\end{aligned}
$$

This function has an analytic extension in the finite lower half plane, given by

$$
F^{\ell}(z)=F(z)-\frac{2 \pi i}{E} e^{-\frac{2 z^{2}}{E^{2}}}
$$

We find, numerically, for a reasonable choice of parameters and a simple assumption for $\varphi(x)$, that the analytic continuation of the function

$$
\begin{aligned}
(\varphi|G(z)| \varphi) & =\int d x d x^{\prime} \varphi^{*}(x)\left(\frac{1}{z+E x} \delta\left(x-x^{\prime}\right)\right. \\
& \left.+\lambda \sqrt{\frac{2}{\pi}} \frac{e^{-x^{2}} e^{-x^{\prime 2}}}{(z+E x)\left(z+E x^{\prime}\right)} \frac{1}{1-\lambda \sqrt{\frac{2}{\pi}} F(z)}\right) \varphi(x),
\end{aligned}
$$

defined by (2.8) and (2.11), into the lower half plane, has a pole, inducing an exponential decay term to the amplitude. The function $(\varphi|G(z)| \varphi)$ has a very simple form if we assume that $\varphi(x)$ has the Gaussian form

$$
\varphi(x)=\sqrt{\frac{2}{\pi}} e^{-x^{2}} .
$$


The first term of (2.12) contains $e^{-2 x^{2}}$; its integral with the denominator $z+E x$ is, according to $(2.7)$, the function $F(z)$. The second term factorizes into two integrals of the same form. It then follows, with this assumption on $\varphi$, that

$$
\begin{aligned}
(\varphi|G(z)| \varphi) & =\sqrt{\frac{2}{\pi}} F(z)+\lambda\left(\frac{2}{\pi}\right) \frac{(F(z))^{2}}{1-\lambda \sqrt{\frac{2}{\pi}} F(z)} \\
& =\frac{\sqrt{\frac{2}{\pi}} F(z)}{1-\lambda \sqrt{\frac{2}{\pi}} F(z)} .
\end{aligned}
$$

The analytic continuation of this function into the lower half plane is achieved by the continuation of $F(z)$ to $F^{\ell}(z)$; this function has no poles in the finite lower half plane, and hence the pole can only come from the condition

$$
g(z) \equiv 1-\lambda \sqrt{\frac{2}{\pi}} F^{\ell}(z)=0 .
$$

For the value $\lambda / E=11$, MAPLE provides us with a unique solution for the position of the pole, $z_{0}=-4.446-.31896 \times 10^{-15} i$, which has, as expected, a very small imaginary part for this reasonably physical choice of parameters. Differentiating (2.15) implicitly with respect to $E$, we find that the real part of the pole moves to more negative values as $E$ increases. Since the unpertubed system has mean position of the particle at zero, this shift corresponds to an increase in field induced polarization with increasing value of the field.

There remains, however, a contribution to the survival amplitude from integration on a line running from $+\infty$ to $-\infty$ on the real part of $z=\xi+i \zeta$, where $\zeta$ can be very large and negative. The contribution of this so-called background integral is strongly suppressed by the exponent $\exp (-i z t)$ for $t$ large and positive. For small $t$, however, this suppression is not strong unless $\zeta \rightarrow-\infty$. However, in this limit, the integrand is not well-defined, since for any large and negative $\zeta$, the contribution of the discontinuity in $F(z)$ strongly suppresses the integrand for $\xi$ small compared to $\zeta$. This suppression is not maintained, however, for the contributions from $\xi$ in the neighborhood or greater than $\zeta$. Hence the convergence of the contribution on the background is not uniform. We see from the general argument (1.2) that the pole contribution cannot represent the result precisely for small $t$, and therefore the Wigner-Weisskopf treatment, even in this case of unbounded spectrum, cannot result in a pure exponential (semigroup) behavior for the reduced evolution. It is exactly in this respect that the Lax-Phillips treatment provides a result which is closer to the physics of irreversible decay.

\section{Lax-Phillips theory}

The Stark effect admits a simple transition to the Lax-Phillips framework, since the Hamiltonian is unbounded. In the following, we briefly describe the theory of Lax and Phillips $^{9}$ and its extension to the quantum case ${ }^{13}$, and then apply the formalism to the model we are using. 
The scattering theory of Lax and Phillips assumes the existence of a Hilbert space $\overline{\mathcal{H}}$ of physical states in which there are two distinguished orthogonal subspaces $\mathcal{D}_{+}$and $\mathcal{D}_{-}$ with the properties

$$
\begin{array}{rlr}
U(\tau) \mathcal{D}_{+} & \subset \mathcal{D}_{+} & \tau>0 \\
U(\tau) \mathcal{D}_{-} & \subset \mathcal{D}_{-} & \tau<0 \\
\bigcap_{\tau} U(\tau) \mathcal{D}_{ \pm} & =\{0\} & \\
\bigcup_{\tau} U(\tau) \mathcal{D}_{ \pm} & =\overline{\mathcal{H}},
\end{array}
$$

i.e., the subspaces $\mathcal{D}_{ \pm}$are stable under the action of the full unitary dynamical evolution $U(\tau)$, a function of the physical laboratory time, for positive and negative times $\tau$ respectively; over all $\tau$, the evolution operator generates a dense set in $\overline{\mathcal{H}}$ from either $\mathcal{D}_{+}$or $\mathcal{D}_{-}$. We shall call $\mathcal{D}_{+}$the outgoing subspace and $\mathcal{D}_{-}$the incoming subspace with respect to the group $U(\tau)$.

A theorem of Sinai $^{20}$ then assures that $\overline{\mathcal{H}}$ can be represented as a family of Hilbert spaces obtained by foliating $\overline{\mathcal{H}}$ along the real line, which we shall call $\{s\}$, in the form of a direct integral

$$
\overline{\mathcal{H}}=\int_{\oplus} \mathcal{H}_{s}
$$

where the set of auxiliary Hilbert spaces $\mathcal{H}_{s}$ are all isomorphic. Representing these spaces in terms of square-integrable functions, we define the norm in the direct integral space (we use Lesbesgue measure) as

$$
\|f\|^{2}=\int_{-\infty}^{\infty} d s\left\|f_{s}\right\|_{H}^{2},
$$

where $f \in \bar{H}$ represents a vector in $\overline{\mathcal{H}}$ in terms of the $L^{2}$ function space $L^{2}(-\infty, \infty, H)$, and $f_{s} \in H$, the $L^{2}$ function space representing $\mathcal{H}_{s}$ for any $s$. The Sinai theorem furthermore asserts that there are representations for which the action of the full evolution group $U(\tau)$ on $L^{2}(-\infty, \infty, H)$ is translation by $\tau$ units. Given $D_{ \pm}$(the $L^{2}$ spaces representing $\left.\mathcal{D}_{ \pm}\right)$, there is such a representation, called the incoming translation representation ${ }^{1}$, for which functions in $D_{-}$have support in $L^{2}(-\infty, 0, H)$, and another called the outgoing translation representation, for which functions in $D_{+}$have support in $L^{2}(0, \infty, H)$.

Lax and Phillips ${ }^{9}$ show that there are unitary operators $W_{ \pm}$, called wave operators, which map elements in $\overline{\mathcal{H}}$, respectively, to these representations. They define an $S$-matrix,

$$
S=W_{+} W_{-}^{-1}
$$

which connects these representations; it is unitary, commutes with translations, and maps $L^{2}(-\infty, 0)$ into itself*. The singularities of this $S$-matrix, in what we shall define as the

* Note that the $S$ matrix of the Lax-Phillips theory maps the incoming to the outgoing representations unlike the $S$ matrix of the usual scattering theory which maps incoming to outgoing states 
spectral representation, correspond to the spectrum of the generator of the exact semigroup characterizing the evolution of the unstable system.

With the assumptions stated above on the properties of the subspaces $\mathcal{D}_{+}$and $\mathcal{D}_{-}$, Lax and Phillips ${ }^{9}$ prove that the family of operators

$$
Z(\tau) \equiv P_{+} U(\tau) P_{-} \quad(\tau \geq 0)
$$

where $P_{ \pm}$are projections into the orthogonal complements of $\mathcal{D}_{ \pm}$, respectively, is a contractive, continuous, semigroup satisfying

$$
Z\left(\tau_{1}\right) Z\left(\tau_{2}\right)=Z\left(\tau_{1}+\tau_{2}\right)
$$

This operator annihilates vectors in $\mathcal{D}_{ \pm}$and carries the space

$$
\mathcal{K}=\overline{\mathcal{H}} \ominus \mathcal{D}_{+} \ominus \mathcal{D}_{-}
$$

into itself, with norm tending to zero for every element in $\mathcal{K}$. One may demonstrate the semigroup property by noting that the operator acting on a vector in $D_{-}$vanishes by definition; on a vector in $D_{+}$, it vanishes due to the invariance of $D+$. The scalar product on the left with a vector $g$ in $D_{+}$vanishes by definiton; if $g$ is in $D_{-}$, the factor $P_{+}$may be suppressed, since such a $g$ is in a subspace othogonal to $D_{+}$. Hence the operator (3.5) can only connect $\mathcal{K}$ to $\mathcal{K}$. In the product

$$
P_{+} U\left(\tau_{1}\right) P_{-} P_{+} U\left(\tau_{2}\right) P_{-}
$$

one may suppress the factor $P_{-}$following $P_{+}$between the two unitaries since vectors in $\mathcal{K}$ are orthogonal to $D_{-}$. Then, consider

$$
P_{+} U\left(\tau_{1}\right) P_{+} U\left(\tau_{2}\right)=P_{+} U\left(\tau_{1}\right)\left[1-\left(1-P_{+}\right)\right] U\left(\tau_{2}\right)
$$

the second term in square brackets makes no contribution since it is a projection into $D_{+}$, and $U\left(\tau_{1}\right)$ leaves this subspace invariant. The composition of $U\left(\tau_{1}\right)$ and $U\left(\tau_{2}\right)$ then proves (3.6).

The outgoing subspace $D_{+}$is defined, in the outgoing representation, in terms of support properties (this is also true for the incoming subspace in the incoming representation). One can then easily understand that the fundamental difference between Lax-Phillips theory and the standard quantum theory lies in this property. The subspace defining the unstable system in the standard theory is usually defined as the eigenstate of an unperturbed Hamiltonian, and is not associated with an interval on a line. The subspaces of the Lax-Phillips theory are associated with intervals (i.e., the positive and negative half-lines in the outgoing and incoming representations). It was shown in ref. 11, for a Lax-Phillips theory constructed on a model of free evolution, that the generator of the motion restricted to the subspace $\mathcal{K}$ is symmetric but not self-adjoint. To briefly review this point in a slightly different way, consider the infinitesimal generator of the semigroup. According to the Stone theorem, the unitary evolution $U(\tau)$ can be represented as $e^{-i K \tau}$; since 
$P_{+} U(\tau) P_{-}$takes $\mathcal{K}$ into itself, as shown by Lax and Phillips, $P_{+} U(\tau) P_{-}=P_{\mathcal{K}} U(\tau) P_{\mathcal{K}}$, and therefore the infinitesimal generator (the derivative at $\tau=0$ ) satisfies

$$
P_{+} K P_{-}=P_{\mathcal{K}} K P_{\mathcal{K}}
$$

Consider vector-valued functions $f(s), g(s)$ in $\mathcal{K}$, in the translation representation, so that (we may suppress $P_{-}$acting on $f$ or $g$ )

$$
\left(P_{+} K\right) f(s)=-i \theta(-s) \frac{\partial}{\partial s} f(s) .
$$

We then take the scalar product with $g$ to obtain

$$
\begin{aligned}
\int_{-\infty}^{\infty} g^{*}(s)\left(P_{+} K f\right)(s) d s & =-i \int g^{*}(s) \theta(-s) \frac{\partial f(s)}{\partial s} d s \\
& =-i g^{*}(0) f(0)+\int\left(-i \theta(-s) \frac{\partial g(s)}{\partial s}\right)^{*} f(s) d s \\
& =-i g(0)^{*} f(0)+\int\left(P_{+} K g\right)^{*}(s) f(s) d s,
\end{aligned}
$$

so that the operator $P_{+} K P_{-}=P_{\mathcal{K}} K P_{\mathcal{K}}$ is not self-adjoint. It is through this mechanism, that the subspaces are defined by (semibounded) support properties on a variable acted upon by an operator represented as a derivative in the translation representation (somewhat analogous to the non-self-adjointness of the operator $i \partial / \partial r$ in three dimensional spherical coordinates), that the Lax-Phillips theory provides a description that has the semigroup property for the evolution of an unstable system.

It follows immediately from the property of a contractive semigroup that the generator has, in fact, a family of complex eigenvalues in the lower half-plane; the eigenfunctions are ${ }^{9}$

$$
f_{\mu}(s)= \begin{cases}e^{\mu s} n, & s \leq 0 \\ 0, & s>0\end{cases}
$$

where $n$ is some vector in the auxiliary space.

In order to construct an imbedding of Lax-Phillips theory into the quantum theory ${ }^{13}$ we assume that there exist wave operators $\Omega_{ \pm}$which intertwine this dynamical operator with an unperturbed dynamical operator $K_{0}$. We shall assume that $K_{0}$ has absolutely continuous spectrum in $(-\infty, \infty)$.

We begin the development of the quantum Lax-Phillips theory with the construction of the incoming and outgoing translation representations. In this way, we shall construct explicitly the foliations required by the Lax-Phillips theory described above ${ }^{13}$. Let us define the free spectral representation ${ }^{9}$ in terms of the Fourier transform

$$
{ }_{f}\langle\sigma \beta \mid g\rangle=\int e^{-i \sigma s}\langle s \beta \mid g\rangle d s,
$$

where ${ }_{f}\langle\sigma \beta \mid g\rangle$ satisfies

$$
{ }_{f}\left\langle\sigma \beta\left|K_{0}\right| g\right\rangle=\sigma_{f}\langle\sigma \beta \mid g\rangle .
$$


Here $|g\rangle$ is an element of $\overline{\mathcal{H}}$ and $\beta$ corresponds to the variables (measure space) of the auxiliary space associated to each value of $\sigma$, which, with $\sigma$, comprise a complete spectral set. The functions may be thought of as a set of functions of the variables $\beta$ indexed on the variable $\sigma$ in a continuous sequence of auxiliary Hilbert spaces isomorphic to $H$. Clearly, $K_{0}$ acts as the generator of translations in the representation ${ }_{f}\langle s \beta \mid g\rangle$.

We now proceed to define the incoming and outgoing subspaces $\mathcal{D}_{ \pm}$.

Let us consider the sets of functions with support in $L^{2}(0, \infty)$ and in $L^{2}(-\infty, 0)$, and call these subspaces $D_{0}^{ \pm}$. The Fourier transform back to the free spectral representation provides the two sets of Hardy class functions

$$
{ }_{f}\left\langle\sigma \beta \mid g_{0}^{ \pm}\right\rangle=\int e^{-i \sigma s}\left\langle s \beta \mid g_{0}^{ \pm}\right\rangle d s \in H_{ \pm},
$$

for $g_{0}^{ \pm} \in D_{0}^{ \pm}$.

We may now define the subspaces $\mathcal{D}_{ \pm}$in the Hilbert space of states $\overline{\mathcal{H}}$. To do this we first map these Hardy class functions in $\bar{H}$ to $\overline{\mathcal{H}}$, i.e., we define the subspaces $\mathcal{D}_{0}^{ \pm}$by

$$
\int \sum_{\beta}|\sigma \beta\rangle_{f}\left\langle\sigma \beta \mid g_{0}^{ \pm}\right\rangle d \sigma \in \mathcal{D}_{0}^{ \pm} .
$$

We shall assume that there are wave operators which intertwine $K_{0}$ with the full evolution $K$, i.e., that the limits

$$
\lim _{\tau \rightarrow \pm \infty} e^{i K \tau} e^{-i K_{0} \tau}=\Omega_{ \pm}
$$

exist on a dense set in $\overline{\mathcal{H}}$. The conditions for the existence of such wave operators are satisifed if the difference between $K$ and $K_{0}$ is a "small" operator. We shall explicitly construct these wave operators in the case of the Stark effect that we study here.

The construction of $\mathcal{D}_{ \pm}$is then completed with the help of the wave operators. We define these subspaces by

$$
\begin{aligned}
& \mathcal{D}_{+}=\Omega_{+} \mathcal{D}_{0}^{+} \\
& \mathcal{D}_{-}=\Omega_{-} \mathcal{D}_{0}^{-} .
\end{aligned}
$$

We remark that these subspaces are not produced by the same unitary map. This procedure is necessary to realize the Lax-Phillips structure non-trivially; if a single unitary map were used, then there would exist a transformation into the space of functions on $L^{2}(-\infty, \infty, H)$ which has the property that all functions with support on the positive half-line represent elements of $\mathcal{D}_{+}$, and all functions with support on the negative half-line represent elements of $\mathcal{D}_{-}$in the same representation; the resulting Lax-Phillips $S$-matrix would then be trivial. The requirement that $\mathcal{D}_{+}$and $\mathcal{D}_{-}$be orthogonal is not an immediate consequence of our construction. Since the functions ${ }_{f}\left\langle s \beta \mid g_{0}^{ \pm}\right\rangle$have support in, respectively, the positive and negative half lines, and the orthogonality of $\mathcal{D}_{ \pm}$is determined by the integral of the product of these functions with an operator valued kernel $\mathbf{S}\left(s-s^{\prime}\right)$ (to be defined below), one sees that suitable analyticity properties of the transformed kernel $S(\sigma)$ assure that these 
subspaces will be orthogonal. This analyticity property (upper half plane analyticity) is true in the Stark model that we treat.

The wave operators defined by (3.15) intertwine $K$ and $K_{0}$, i.e.,

$$
K \Omega_{ \pm}=\Omega_{ \pm} K_{0}
$$

we may therefore construct the outgoing (incoming) spectral representations from the free spectral representation. Since

$$
\begin{aligned}
K \Omega_{ \pm}|\sigma \beta\rangle_{f} & =\Omega_{ \pm} K_{0}|\sigma \beta\rangle_{f} \\
& =\sigma \Omega_{ \pm}|\sigma \beta\rangle_{f}
\end{aligned}
$$

we may identify

$$
|\sigma \beta\rangle_{\substack{\text { out } \\ \text { in }}}=\Omega_{ \pm}|\sigma \beta\rangle_{f}
$$

The Lax-Phillips $S$-matrix is defined as the operator, on $\bar{H}$, which carries the incoming to outgoing translation representations of the evolution operator $K$. Suppose $g$ is an element of $\overline{\mathcal{H}}$; its incoming spectral representation, according to $(3.19)$, is

$$
\left.\left.{ }_{i n}\langle\sigma \beta| g\right)={ }_{f}\langle\sigma \beta| \Omega_{-}^{-1} g\right) .
$$

Let us now act on this function with the Lax-Phillips $S$-matrix in the free spectral representation, and require the result to be the outgoing representer of $g$ :

$$
\begin{aligned}
\left.{ }_{\text {out }}\langle\sigma \beta| g\right) & \left.={ }_{f}\langle\sigma \beta| \Omega_{+}^{-1} g\right) \\
& \left.=\int d \sigma^{\prime} \sum_{\beta^{\prime}}{ }_{f}\left\langle\sigma \beta|\mathbf{S}| \sigma^{\prime} \beta^{\prime}\right\rangle_{f}{ }_{f}\left\langle\sigma^{\prime} \beta^{\prime}\right| \Omega_{-}^{-1} g\right)
\end{aligned}
$$

where $\mathbf{S}$ is the Lax-Phillips $S$-operator (defined on $\overline{\mathcal{H}}$ ). Transforming the kernel to the free translation representation with the help of (3.11), i.e.,

$$
{ }_{f}\left\langle s \beta|\mathbf{S}| s^{\prime} \beta^{\prime}\right\rangle_{f}=\frac{1}{(2 \pi)^{2}} \int d \sigma d \sigma^{\prime} e^{i \sigma s} e^{-i \sigma^{\prime} s^{\prime}}\left\langle\sigma \beta|\mathbf{S}| \sigma^{\prime} \beta^{\prime}\right\rangle_{f}
$$

we see that the relation (3.21) becomes, after using Fourier transform in a similar way to transform the in and out spectral representations to the corresponding in and out translation representations,

$$
\begin{aligned}
\left.\left.{ }_{\text {out }}\langle s \beta| g\right)={ }_{f}\langle s \beta| \Omega_{+}^{-1} g\right) & \left.=\int d s^{\prime} \sum_{\beta^{\prime}}\left\langle s \beta|\mathbf{S}| s^{\prime} \beta^{\prime}\right\rangle_{f f}\left\langle s^{\prime} \beta^{\prime}\right| \Omega_{-}^{-1} g\right) \\
& \left.=\int d s^{\prime} \sum_{\beta^{\prime}}\left\langle s \beta|\mathbf{S}| s^{\prime} \beta^{\prime}\right\rangle_{f i n}\left\langle s^{\prime} \beta^{\prime}\right| g\right) .
\end{aligned}
$$

Hence the Lax-Phillips $S$-matrix is given by

$$
S=\left\{{ }_{f}\left\langle s \beta|\mathbf{S}| s^{\prime} \beta^{\prime}\right\rangle_{f}\right\},
$$


in free translation representation. It follows from the intertwining property (3.17) that

$$
{ }_{f}\left\langle\sigma \beta|\mathbf{S}| \sigma^{\prime} \beta^{\prime}\right\rangle_{f}=\delta\left(\sigma-\sigma^{\prime}\right) S^{\beta \beta^{\prime}}(\sigma),
$$

This result can be expressed in terms of operators on $\overline{\mathcal{H}}$. Let

$$
w_{-}^{-1}=\left\{{ }_{f}\langle s \beta| \Omega_{-}^{-1}\right\}
$$

be a map from $\overline{\mathcal{H}}$ to $\bar{H}$ in the incoming translation representation, and, similarly,

$$
w_{+}^{-1}=\left\{{ }_{f}\langle s \beta| \Omega_{+}^{-1}\right\}
$$

a map from $\overline{\mathcal{H}}$ to $\bar{H}$ in the outgoing translation representation. It then follows from (3.23) that

$$
S=w_{+}^{-1} w_{-},
$$

as a kernel on the free translation representation. This kernel is understood to operate on the representer of a vector $g$ in the incoming representation and map it to the representer in the outgoing representation.

\section{Application to the Stark model}

Since the spectrum of the Stark model that we are using has spectrum $(-\infty, \infty)$, we may take for the generator of motion

$$
K=H_{\text {Stark }} ; \quad K_{0}=H_{0 \text { Stark }} ; \quad V=V_{\text {Stark }},
$$

where $H_{\text {Stark }}, H_{0 \text { Stark }}$ and $V=V_{\text {Stark }}$ are the operators defined in Section 2. Since $H_{0}$ is proportional to $x$, we may make use of the canonical commutation relations of the quantum theory to identify the momentum $p$ as proportional to the (foliation) variable of the unperturbed (free) translation representation, i.e., one may take $s=p / E$, implying that $\left[s, K_{0}\right]=i$ (we have taken $\left.\hbar=1\right)$. We then have

$$
\left(e^{-i K_{0} \tau} f\right)(s)=f(s-\tau),
$$

or, in differential form,

$$
\left(K_{0} f\right)(s)=-i \frac{d}{d s} f(s) .
$$

The auxiliary Hilbert spaces of the corresponding Lax-Phillips theory are one-dimensional.

The spectrum of $K_{0}$, given by $\{-E x\}$, with $-\infty<x<\infty$, can then be identified with $\sigma$ of the Lax-Phillips (unperturbed) energy representation. We shall follow this formal identification to develop the Lax-Phillips theory of resonances, and return to the original interpretation of $x$ and $p$ to obtain physical information about the resonant state.

The wave operators are defined as

$$
\Omega_{ \pm}=\lim _{\tau \rightarrow \pm \infty} e^{i K \tau} e^{-i K_{0} \tau} .
$$


We shall calculate the matrix elements of the wave operator in the unperturbed energy representation. It will be convenient, moreover, to use directly the measure on the spectrum of $x$; we therefore use kets of the form $|x\rangle \equiv \sqrt{|E|}|\sigma\rangle$.

Following the standard procedure for taking these limits, one finds for the representation $\{|x\rangle$, that

$$
\left\langle x\left|\Omega_{ \pm}\right| x^{\prime}\right\rangle=\delta\left(x-x^{\prime}\right)-\lim _{\varepsilon \rightarrow 0_{+}}\left\langle x\left|\frac{1}{H+E x^{\prime} \pm i \varepsilon} V\right| x^{\prime}\right\rangle .
$$

Since this formula is bilinear in the kets $|x\rangle$ and $\left|x^{\prime}\right\rangle$, one could use equally well the kets $|\sigma\rangle$ and $\left|\sigma^{\prime}\right\rangle$.

The operator multiplying $V$ in (4.5) is $-G(z)$, for $z=-E x^{\prime} \mp i \varepsilon$; the matrix elements of this operator were evaluated in (2.8). Carrying out the integral for the product $G(z) V$ with the help of the definition (2.7), one finds that

$$
\left\langle x|G(z) V| x^{\prime}\right\rangle=\lambda \sqrt{\frac{2}{\pi}} \frac{e^{-\left(x^{2}+x^{\prime 2}\right)}}{(z+E x)\left(1-\lambda \sqrt{\frac{2}{\pi}} F(z)\right)}
$$

The wave operators are then given by

$$
\left\langle x\left|\Omega_{ \pm}\right| x^{\prime}\right\rangle=\delta\left(x-x^{\prime}\right)+\lambda \sqrt{\frac{2}{\pi}} \frac{e^{-\left(x^{2}+x^{\prime 2}\right)}}{\left(E\left(x-x^{\prime}\right) \mp i \varepsilon\right)\left(1-\lambda \sqrt{\frac{2}{\pi}} F\left(-E x^{\prime} \mp i \varepsilon\right)\right)}
$$

Using a partial fraction decomposition for the product of the $G V$ terms, one easily verifies that the operators $\Omega_{ \pm}$are unitary.

We now turn to the construction of the incoming and outgoing translation representations. To do this, we follow the method of reference 13. We define the free outgoing translation as the set of functions with support in $s$ (i.e., $p / E$ in the Stark model) on the positive real axis. By (3.11), the functions

$$
f_{+}^{0}(x)=\int_{0}^{\infty} e^{i p x} f_{+}^{0}(p) d p \equiv\left\langle x \mid f_{+}^{0}\right\rangle
$$

are in the free outgoing translation representation, and are analytic in the upper half $x$ plane (lower half $\sigma$-plane). Since the wave operators intertwine $K_{0}$ and $K$, functions of the (full) outgoing representation are then given by

$$
\int_{-\infty}^{\infty}\left\langle x^{\prime}\left|\Omega_{+}\right| x\right\rangle d x f_{+}^{0}(x) d x=f_{+}^{\text {out }}\left(x^{\prime}\right) \in D_{+} .
$$

Given a function of the type $f_{+}^{0}(x)$, one can calculate the resulting function $f_{+}^{\text {out }}\left(x^{\prime}\right)$ explicitly by noting that the boundary value of $F(z)$ from below the real axis is

$$
F\left(-E x^{\prime}\right)_{\text {below }}=\lim _{\varepsilon \rightarrow 0_{+}} \int_{-\infty}^{\infty} \frac{e^{-2 x^{\prime \prime 2}}}{E\left(x^{\prime \prime}-x^{\prime}\right)-i \varepsilon} d x^{\prime \prime}=\frac{i \pi}{E} e^{-2 x^{\prime 2}} \operatorname{erfc}\left(-i \sqrt{2} x^{\prime}\right)
$$


For the construction of the incoming translation representation, one uses $\Omega_{-}$and a corresponding set of functions $f_{-}^{0}(x)$ with support on the negative half line. The kernel of integration then contains $F\left(-E x^{\prime}-i \varepsilon\right)$; this may be obtained from (2.10), i.e.,

$$
F\left(-E x^{\prime}\right)_{a b o v e}=F\left(-E x^{\prime}\right)_{b e l o w}-\frac{2 \pi i}{E} e^{-2 x^{\prime 2}} .
$$

We now turn to the calculation of the $S$-matrix. We see from (3.21) that

$$
\left\langle x|\mathbf{S}| x^{\prime}\right\rangle=\left\langle x\left|\Omega+{ }^{-1} \Omega_{-}\right| x^{\prime}\right\rangle .
$$

We now use the definition (3.15) for the wave operators; following the standard method ${ }^{22}$, we find that

$$
\left\langle x|\mathbf{S}| x^{\prime}\right\rangle=\delta\left(x-x^{\prime}\right)\left(1+\frac{2 \pi i}{E} \lim _{\varepsilon \rightarrow 0_{+}}\left\langle x\left|T\left(-E x^{\prime}+i \varepsilon\right)\right| x^{\prime}\right\rangle,\right.
$$

where $T(z) \equiv V(1+G(z) V)$. We now compute

$$
\begin{aligned}
\lim _{\varepsilon \rightarrow 0_{+}}\left\langle x\left|T\left(-E x^{\prime}+i \varepsilon\right)\right| x^{\prime}\right\rangle & =\lim _{\varepsilon \rightarrow 0_{+}} \int_{-\infty}^{\infty} \lambda \sqrt{\frac{2}{\pi}} e^{-x^{2}+x^{\prime \prime 2}}\left[\delta\left(x^{\prime \prime}-x^{\prime}\right)\right. \\
& \left.-\frac{\lambda \sqrt{\frac{2}{\pi}} e^{-\left(x^{\prime 2}+x^{\prime \prime 2}\right)}}{\left(E\left(x^{\prime}-x^{\prime \prime}\right)-i \varepsilon\right)\left(1-\lambda \sqrt{\frac{2}{\pi}} F\left(-E x^{\prime}+i \varepsilon\right)\right.}\right] d x^{\prime \prime} \\
& =\lim _{\varepsilon \rightarrow 0_{+}} \frac{\lambda \sqrt{\frac{2}{\pi}} e^{-\left(x^{2}+x^{\prime 2}\right)}}{1-\lambda \sqrt{\frac{2}{\pi}} F\left(-E x^{\prime}+i \varepsilon\right)}
\end{aligned}
$$

It then follows that

$$
\left\langle x|\mathbf{S}| x^{\prime}\right\rangle=\delta\left(x-x^{\prime}\right)\left[1+\frac{2 \pi i}{E} \lim _{\varepsilon \rightarrow 0_{+}} \frac{\lambda \sqrt{\frac{2}{\pi}} e^{-2 x^{2}}}{1-\lambda \sqrt{\frac{2}{\pi}} F(-E x+i \varepsilon)}\right],
$$

so that we may write

$$
\left\langle x|\mathbf{S}| x^{\prime}\right\rangle \equiv \delta\left(x-x^{\prime}\right) S(x) .
$$

If we write the semigroup evolution, restricted to the subspace $\mathcal{K}$, as

$$
Z(\tau)=e^{-i B \tau},
$$

it follows from the contractive semigroup property that the operator $B$ has an eigenvector in the outgoing representation satisfying

$$
B f_{\text {out }}=\mu f_{\text {out }},
$$


with $\mu$ in the lower half plane, for which the eigenfunctions are of the form (with support in $(0, \infty))$

$$
f_{\text {out }}(s)=e^{-i \mu s} n,
$$

where $n$ is a vector in the auxiliary space (in our Stark model, one dimensional). The eigenfunctions in the outgoing $x$ representation (corresponding to the "energy" variable $\sigma)$, are then of the form

$$
f_{\text {out }}(x)=\frac{i n}{x-\mu}
$$

The $S$-matrix, connecting the incoming to outgoing representations, therefore has the form

$$
S(x) \sim \frac{r}{x-z_{0}},
$$

where $z_{0}$ is the position of the pole of the diagonal $S$-matrix in the lower half plane, which we identify with the semigroup exponent $\mu$, and $r$ is the residue. From (4.15), one sees that the pole of the $S$-matrix corresponds to a zero of the denominator

$$
1-\lambda \sqrt{\frac{2}{\pi}} F(-E x+i \varepsilon)
$$

continued to the lower half plane, i.e., we must find the zero of

$$
1-\lambda \sqrt{\frac{2}{\pi}} F^{\ell}(z) .
$$

This is precisely the pole (zero of $(2.15)$ ) which occurs in the pole approximation of the Wigner-Weisskopf theory discussed in Section 2. The residue of the pole in (4.21) is then given by

$$
r=\frac{8 \pi i}{E^{3}} \lambda \sqrt{\frac{2}{\pi}} \frac{e^{\frac{-2 z_{0}^{2}}{E^{2}}}}{z_{0}-\lambda} .
$$

\section{Conclusions and Discussion}

We have studied a model for resonances which provides the possibility of analysis using the Wigner-Weisskopf method, the standard technique for such analyses, giving exponential behavior in the pole approximation, but also accessible to direct analysis in the Lax-Phillips framework. In the Wigner-Weisskopf analysis, the resonance is described by the position of a pole in the complex energy plane, and does not have a state in the Hilbert space associated with it. Although there has been considerable study of the application of the method of rigged Hilbert spaces ${ }^{23}$, or Gel'fand triples $(D \subset H \subset \bar{H}$, where $\bar{H}$ is defined as the set of bounded linear functionals on the subspace $D$ ), for the description of resonances which satisfies the property of exact exponential decay (without the "background" corrections to the Wigner-Weisskopf pole approximation), the elements

of the Gel'fand triple are not, in general, vectors of a Hilbert space (they belong to a Banach space), and have no scalar products. Hence, it is not possible to compute the 
expectation value of an observable (unless its extension to $\bar{H}$ maps it into the smaller space $D$ on which the linear functionals of $\bar{H}$ are defined), or to study physical properties such as localization of the state.

The Lax-Phillips formulation describes the resonant state as an element of a Hilbert space, and answers to such questions then become accessible. As a simple example, we have computed the resonant state for the Stark model that we have used in (4.20). The variable $x$ used here corresponds to the "energy" in the Lax-Phillips formal structure, but retains its physical meaning in the result as position. Note that this is also true in our formulation of the Wigner-Weisskopf model, taking for the unperturbed Hamiltonian the term $-E x$ in the Hamiltonian (2.1) since this term is large compared to the term producing the imbedded bound state. The position variable occurs in the Hamiltonian producing a continuous energy spectrum $\{E x\}$. The interpretation of the poles of the $S$ matrix, or resolvent therefore remains, as in the usual formulation of resonance problems, as occurring in the complex energy plane, but the variable $x$ retains its physical meaning as coordinate as well. This method leads us to an approximate exponential decay law in time in the pole approximation of Wigner-Weisskopf theory, and to an exact exponential decay law in the Lax-Phillips treatment, with precisely the same exponent.

In the framework of general Lax-Phillips theory, the resonant state carries the pole as in (4.20) where the $x$ that appears there would be replaced by another symbol, say, $\sigma$, associated with unperturbed energy, and the distribution over space of the wave function would reside in the vector $n$ of the auxiliary Hilbert space. In our case, this vector is just a number (one-dimensional), and the space distribution is provided by the equivalence of (unperturbed) energy and the variable $x$.

The resonance state provided by the Lax-Phillips theory in the "energy" representation actually therefore corresponds to a distribution of $x$ values in the resonant state.

$$
\left|f_{\text {out }}\right|^{2}=\frac{\|n\|^{2}}{\left(x-R e z_{0}\right)^{2}+\left|I m z_{0}\right|^{2}},
$$

a Cauchy distribution with width $\left|\operatorname{Im} z_{0}\right|$. The Cauchy distribution is centered on $R e z_{0}$, corresponding to a shift away from the mean value of $x=0$ in the bound state in the absence of electric field; as we have seen, the pole moves further to the left with increasing field, so that the center of the wave packet moves to the left.

\section{References}

1. V.F. Weisskopf and E.P. Wigner, Zeits. f. Phys. 63, (1930); 65, 18 (1930).

2. B. Misra and E.C.G. Sudarshan, Jour. Math. Phys. 18, 756 (1977). For more recent discussion and references, see P. Facchi and S. Pascazio, "Unstable Systems and Quantum Zeno Phenomena in Quantum Field Theory," quant-ph/0202127.

3. N. Bleistein, R. Handelsman, L.P. Horwitz and H. Neumann, Nuovo Cimento 41A, 389 (1977).

4. L.P. Horwitz and J.-P. Marchand, Helv. Phys. Acta 42, 1039 (1969); L.P. Horwitz and J.-P. Marchand, Rocky Mountain Journa;l of Mathematics 1, 225 (1971).

5. C. Piron, Foundations of Quantum Physics, W.A. Benjamin, Inc., Reading, Mass. (1967); see also discussions in ref. 11. 
6. B. Winstein, et al, Results from the Neutral Kaon Program at Fermilab's Meson Center Beamline 1985-1997, published on behalf of the E731, E773 and E799 Collaborations, Fermi National Laboratory, P.O. Box 500, Batavia, Illinois.

7. T.D. Lee, R. Oehme and C.N. Yang, Phys. Rev. 106, 340 (1957); T.T. Wu and C.N. Yang, Phys. Rev, Lett. 13, 380 (1964).

8. L.P. Horwitz and L. Mizrachi, Nuovo Cimento 21A, 625(1974); E. Cohen and L.P. Horwitz, Hadronic Jour. 24593 (2001); see also hep-th/9808030, hep-ph/9811332.

9. P.D. Lax and R.S. Phillips, Scattering Theory, Academic Press, N.Y. (1967).

10. C. Flesia and C. Piron, Helv. Phys. Acta 57, 697 (1984).

11. L.P. Horwitz and C. Piron, Helv. Phys. Acta 66, 694 (1993).

12. E. Eisenberg and L.P. Horwitz, in Advances in Chemical Physics vol.XCIX, p. 245, ed. I. Prigogine and S. Rice, Wiley, New York (1997).

13. L.P. Horwitz and Y.Strauss, in Irreversibility and Causality, Semigroups and Rigged Hilbert Spaces, ed. A. Böhm, H.-D. Doebner and P. Kielanowski, Springer Verlag, New York (1996).

14. Y. Strauss, Int. Jour. Theor. Phys. 42, 2285 (2003).

15. W. Baumgartel, Math. Nachr. 69, 107 (1975); L.P. Horwitz and I.M. Sigal, Helv. Phys. Acta 51, 685 (1978); G. Parravicini, V. Gorini and E.C.G. Sudarshan, J. Math. Phys. 21, 2208 (1980); A. Bohm, Quantum Mechanics: Foundations and Applications, Springer, Berlin (1986); A. Bohm, M. Gadella and G.B. Mainland, Am. J. Phys. 57, 1105 (1989); T. Bailey and W.C. Schieve, Nuovo Cimento 47A, 231 (1978).

16. O. Agam, N.S. Wingreen, B.L. Altshuler, D.C. Ralph, and M. Tinkham, condmat/9611115; O. Agam, B.L. Altshuler and A.V. Andreev, cond-mat/9509102; B. Altshuler, From Anderson Localization to Quantum Chaos, Lecture at the Institute for Advanced Study, Princeton, N.J., 7 February, 1996.

17. E.C.G. Stueckelberg, Helv. Phys. Acta 14, 322, 588 (1941); J. Schwinger, Phys. Rev. 82, 664 (1951); R.P. Feynman, Rev. Mod. Phys. 20, 367 (1948) and Phys. Rev. 80, 440 (1950); L.P. Horwitz and C. Piron, Helv. Phys. Acta 46, 316 (1973); R. Fanchi, Phys. Rev D20,3108 (1979); A. Kyprianides, Phys. Rep. 155, 1 (1986) (and references therein).

18. Y. Strauss and L.P. Horwitz, Found. Phys. 30, 653 (2000).

19. K.O. Friedrichs and P.A. Rejto, Comm. Pure and Appl. Math. 15, 219 (1962).

20. I.P. Cornfield, S.V. Formin and Ya. G. Sinai, Ergodic Theory, Springer, Berlin (1982).

21. Y. Strauss, E. Eisenberg and L.P. Horwitz, Jour. Math. Phys. 41, 8050 (2000).

22. For example, J.R. Taylor, Scattering Theory, John Wiley and Sons, N.Y. (1972); R.J. Newton, Scattering Theory of Particles and Waves, McGraw Hill, N.Y. (1976). 